\title{
Some recent results on the stability of linear time varying systems
}

\author{
S PRADEEP and SHASHI K SHRIVASTAVA
}

Department of Aerospace Engineering, Indian Institute of Science, Bangalore 560 012, India

MS received 18 November 1987; revised 6 August 1988

\begin{abstract}
Some theorems derived recently by the authors on the stability of multidimensional linear time varying systems are reported in this paper. To begin with, criteria based on Liapunov's direct method are stated. These are followed by conditions on the asymptotic behaviour and boundedness of solutions. Finally, $L_{2}$ and $L_{\infty}$ stabilities of these systems are discussed. In conclusion, mention is made of some of the problems in aerospace engineering to which these theorems have been applied.
\end{abstract}

Keywords. Stability; linear time varying systems; Liapunov's direct method.

\section{Introduction}

Mathematical equations describing physical systems could be classified, in the order of increasing complexity, into

- linear time invariant (LTI);

- nonlinear time invariant (NLTI);

- linear time variant (LTV);

- nonlinear time variant (NLTV).

Most of these equations are not amenable to closed form solution. Of the aforementioned categories, closed form solutions in terms of the existing elementary functions exist only for LTI systems. Nonavailability of explicit solutions was the primary motivation for the development of the qualitative theory of differential equations, which determine the properties of solutions without explicitly solving the equations.

The present study was motivated by the recent trend towards building very large, complex space structures which require precise control. During deployment of large structures from space stations/platforms, the system parameters vary rapidly and arbitrarily. The equations encountered are typically of the form

$$
M(t) q^{\prime \prime}(t)+G(t) q^{\prime}(t)+K(t) q(t)=P(t) w(t),
$$


where primes denote differentiation with respect to time $t$, the independent variable; $q$ is the $(n \times 1)$ state vector; $w$ is the $(m \times 1)$ input vector; $M\left(=M^{T}>0\right), G$ and $K$ are $(n \times n)$ real matrices, whose elements are functions of time and differentiable; $P$ is an $(n \times m)$ matrix, whose elements are functions of $t$ and differentiable; and $n$ is the dimension of the system, which is often large.

For mechanical systems, the symmetric and skew-symmetric parts of $G$ correspond to damping and gyroscopic forces; and the symmetric and skewsymmetric parts of $K$ correspond to stiffness and circulatory forces. $P$ contains the control gains. Similar equations are also encountered in other disciplines of engineering, economics, ecology, biosystems and demography.

The stability of the system during such an operation is of paramount importance to the mission. Most of the existing stability criteria for time varying systems deal with equations of the form

$$
x^{\prime}(t)=B(t) x(t)+R(t) v(t) .
$$

To apply one of these criteria, (1) has to be converted into (2). This requires inversion and multiplication of matrices, both of which, in addition to being difficult if not impossible for large-dimensioned time varying systems, often lead to a loss of physical insight into the effect of the system parameters on stability. The problems of inversion and multiplication can be circumvented if (1) is converted into the following modified first-order form,

$$
A(t) x^{\prime}(t)=B(t) x(t)+R(t) v(t)
$$

In the analyses carried out by the authors, (i) some new stability theorems have been derived for (1) and (3), and (ii) some of the existing criteria have been modified to deal with such equations.

\section{Liapunov stability}

Very few authors have analysed (1) directly. To our knowledge, there are only two texts completely devoted to the stability of multidimensional linear second-order systems (Lancaster 1966; Huseyin 1978), both of which treat constant coefficient systems. The importance of treating second-order systems as such is evident from the extensive bibliography contained in these two books.

For single degree of freedom linear second-order time varying systems, the Sonin-Polya theorem provides the nature of solutions over a finite interval. It is stated below (Kreider et al 1968).

Sonin-Polya theorem: Let $p(x)>0$ and $q(x) \neq 0$ be continuously differentiable on an interval $I$ and suppose $p(x) q(x)$ is nonincreasing (nondecreasing) on $I$. Then the absolute values of the relative maxima and minima of every nontrivial solution of the equation

$$
\left[p(x) y^{\prime}\right]^{\prime}+q(x) y=0,
$$

are nondecreasing (nonincreasing) as $x$ increases.

In this theorem, (i) stability conditions are imposed directly on the system parameters $p(x)$ and $q(x)$, (ii) these conditions need to be verified only on the interval $I$, and not on the entire semi-infinite time axis, and (iii) are easy to verify. 
Hartman (Coppel 1965) extended the Sonin-Polya theorem to a limited class of multidimensional systems. Later, Shrivastava (1981) extended the Sonin-Polya theorem to encompass a much wider class. It reads:

Theorem 1: Let $A_{0}(x), A_{2}(x)$ be nonsingular and continuously differentiable matrices on an interval $I$. If there exists a positive definite symmetric matrix $S(x)$ such that $\left(S A_{0}^{-1} A_{2}\right)$ is positive definite symmetric (i.e., $A_{0}^{-1} A_{2}$ is symmetrizable),

$$
S^{\prime} \leqslant 0 \text { and } A=\left[S A_{0}^{-1} A_{1}+\left(S A_{0}^{-1} A_{1}\right)^{T}-\left(S A_{0}^{-1} A_{2}\right)^{\prime}\right] \geqslant 0 \text {, }
$$

then the region of possible solutions of the coupled nonautonomous equations,

$$
A_{2}(x) z^{\prime \prime}(x)+A_{1}(x) z^{\prime}(x)+A_{0}(x) z(x)=0,
$$

is-nonincreasing as $x$ increases. If $A>0$, the region will decrease asymptotically.

In the above equation, $z$ is a vector of dependent variables and primes indicate derivatives with respect to the independent variable $x$. Shrivastava (1981) also contains another version of this theorem. Recently, the authors derived several theorems on the stability (Shrivastava \& Pradeep 1985; Pradeep 1987) of secondorder systems using Liapunov's direct method. A typical theorem of this category reads:

Theorem 2: Systems governed by the equation,

$$
M(t) q^{\prime \prime}(t)+\left[G_{1}(t)+G_{2}(t)\right] q^{\prime}(t)+K_{1}(t) q(t)=0,
$$

where $M, G_{1}, G_{2}, K_{1}$ are $(n \times n)$ real matrices whose elements are functions of $t$ and differentiable,

$$
G_{1}=G_{1}^{T}, G_{2}=-G_{2}^{T}, \text { and } K_{1}=K_{1}^{T},
$$

are stable if

(i) $K_{1}>0$, (ii) $M^{\prime} \leqslant 0$, (iii) $2 M K_{1}^{-1}\left(G_{1}+G_{2}\right)-\left(M K_{1}^{-1} M\right)^{\prime} \geqslant 0$, for all $t \in[0, \infty)$.

This theorem was proved by using the Liapunov function

$$
V=(q, M q)+\left(q^{\prime}, M K_{1}^{-1} M q^{\prime}\right) \text {. }
$$

The above conditions were arrived at by ensuring the positive definiteness of $V$ and the negative semi-definiteness of $V^{\prime}$.

It may be noted that (4) includes only systems with a symmetric $K_{1}$. The following corollary of the above theorem is applicable to systems which include a skew-symmetric matrix $K_{2}$ (circulatory forces) along with the symmetric matrix $K_{1}$.

Corollary 2.1: For systems governed by the equation,

$$
M(t) q^{\prime \prime}(t)+G(t) q^{\prime}(t)+K(t) q(t)=0,
$$

where $G$ and $K$ are general time varying matrices, if there exists a symmetric positive definite matrix $S(t)$ such that $S M^{-1} K=\left(S M^{-1} K\right)^{T}$, then a set of sufficient conditions for the stability of (7) is
(i) $S M^{-1} K>0$,
(ii) $S^{\prime} \leqslant 0$,
(iii) $2 S K^{-1} G-\left(S K^{-1} M\right)^{\prime} \geqslant 0$,
for all $t \in[0, \infty)$. 
Theorem 3: Systems governed by the equation,

$$
M(t) q^{\prime \prime}(t)+\left[G_{1}(t)+G_{2}(t)\right] q^{\prime}(t)+K_{1}(t) q(t)=0,
$$

are stable if

$$
\text { (i) } K_{1}>0 \text {, (ii) } K_{1}^{\prime} \leqslant 0 \text {, (iii) } 2 G_{1}-M^{\prime} \geqslant 0 \text {, for all } t \in[0, \infty) \text {. }
$$

These theorems generalize many of the existing theorems on multidimensional linear second-order systems. Their two major shortcomings are that (i) they provide only sufficient conditions (which is also true of the Sonin-Polya theorem), and (ii) though they are easy to apply to systems with symmetric stiffness matrices, they are quite involved for systems with circulatory forces. For circulatory systems, corollary $2 \cdot 1$ shows that a matrix $S$ that symmetrizes the stiffness matrix must be found. There is no general procedure to generate this matrix. Construction of the $S$ matrix has to proceed by trial and error, requiring considerable ingenuity and labour. The following lemma was proved (Shrivastava \& Pradeep 1985; Pradeep 1987) to circumvent determination of the $S$ matrix.

Lemma 1: The bilinear form

$$
U(x, y)=(x, A x)+2(x, B y)+(y, C y),
$$

is positive definite if and only if

$$
\left[\begin{array}{ll}
A & B \\
B^{T} & C
\end{array}\right]
$$

is positive definite.

Some theorems were proved using this lemma. A representative result is the following.

Theorem 4: Systems governed by the equation,

$$
M q^{\prime \prime}+\left(G_{1}+G_{2}\right) q^{\prime}+\left(K_{1}+K_{2}\right) q=0,
$$

are stable if

(i) $K_{1}>0$, and

(ii) $\left[\frac{-M^{\prime}}{\left(M K_{1}^{-1} K_{2}\right)} \mid \frac{\left(M K_{1}^{-1} K_{2}\right)^{T}}{2 M K_{1}^{-1}\left(G_{1}+G_{2}\right)-\left(M K_{1}^{-1} M\right)^{\prime}}\right] \geqslant 0$,

for all $t \in[0, \infty)$.

This does not require determination of the $S$-matrix. However, it requires testing a matrix of twice the original dimension for sign definiteness. It also involves inversion of a matrix.

Instability theorems have also been proved (Shrivastava \& Pradeep 1985; Pradeep 1987) using Liapunov's method. One such instability theorem is given below.

Theorem 5: Systems governed by the equation,

$$
M q^{\prime \prime}+G_{1} q^{\prime}+\left(K_{1}+K_{2}\right) q=0
$$


are unstable if

$$
G_{1}^{\prime}-2 K_{1}-M^{\prime \prime}>0, \text { for all } t \in[0, \infty) .
$$

This theorem is applicable to nongyroscopic systems. For gyroscopic systems, a symmetric positive definite matrix $S_{2}$ must be found to symmetrize $\left(G_{1}+G_{2}\right)$. In order to circumvent this, another theorem has been proved using lemma 1 .

Some of the theorems just stated involve inversion and multiplication of matrices. At the outset, we had expressed our desire to eliminate these operations. However, these theorems are new and are retained in the hope that they may be applicable to systems in which these operations are easy to carry out. The theorems presented here impose conditions directly on the system parameters and do not necessitate transformation to first order. This should prove helpful to analysts and designers dealing with such systems.

\section{Asymptotic behaviour and boundedness of solutions}

For many physical systems, the variation of parameters at large values of time is rather small, making it possible to treat them as almost constant coefficient systems. The following definition (Bellman 1953) quantifies this concept.

Definition: The coefficient matrix $A(t)$ of the differential equation $\mathrm{d} x / \mathrm{d} t=A(t) x$ is called almost constant if

$$
\lim _{t \rightarrow \infty} A(t)=A_{1}, \quad \text { a constant matrix. }
$$

Many theorems exist in literature dealing with the asymptotic behaviour and boundedness of almost constant coefficient systems of the form $x^{\prime}(t)=$ $[B+D(t)] x(t)$ ( $x$ is an $n$-vector, $B$ and $D$ are $n \times n$ matrices), where $B+D(t) \rightarrow E$, a constant matrix as $t \rightarrow \infty$. Most of these results are summarized in well-known texts (Bellman 1953; Cesari 1963; Coppel 1965).

To apply these results to second-order systems described by (1), it must be converted into the first-order form. The difficulties associated with this conversion are the inversion of $M$ and subsequent multiplication with parameter matrices as mentioned earlier, and more important, that the operation of inversion does not preserve the desired form (i.e. each term being the sum of constant and time varying parts). Hence the second order system must be treated as such, or the transformed version (3) should be used. The second-order system can be analysed as such only if the solution of (1) can be written in terms of the solution of the corresponding constant coefficient system (defined as the system obtained by making all the time varying coefficients zero). Since this has not been possible, results are given here for

$$
[A+C(t)] x^{\prime}=[B+D(t)] x .
$$

The results in this section rely on the Bellman-Gronwall lemma (Gronwall 1918; Bellman 1943). The proofs of the theorems (Pradeep 1987; Pradeep \& Shrivastava 1987a) are obtained by first converting the differential equation into a Volterra type matrix integral equation, and then into an inequality by taking the norms of both sides, in a form suitable for application of the lemma. The final results are obtained on its application.

A theorem on the asymptotic behaviour of solutions is as follows. 
Theorem 6: Let $B$ be a constant matrix and $C(t)$ and $D(t)$ be matrices whose elements are continuous functions of $t$. If all solutions of the system

$$
x^{\prime}=B x
$$

approach zero as $t \rightarrow \infty$, at the rate

$$
\|\Phi(t)\| \leqslant m e^{-u t}
$$

where $\Phi$ is the state transition matrix for (15) and if

$$
\|B\|=b,\|C(t)\| \leqslant c<1,\|D(t)\| \leqslant d,\left\|C^{\prime}(t)\right\| \leqslant f,
$$

and these numbers are related by

$$
m(f+b c+d)<u(l-c),
$$

then all solutions of the system

$$
[I+C(t)] x^{\prime}=[B+D(t)] x
$$

approach zero as $t \rightarrow \infty$.

This theorem has been extended further to include a more generalized form of the governing equation

$$
[A+C(t)] x^{\prime}=[B+D(t)] x .
$$

These theorems generalize a theorem stated in Cesari (1963).

A theorem (Pradeep 1987; Pradeep \& Shrivastava 1987a) on the boundedness of solutions, which is an extension of the Dini Hukuhara theorem (Bellman 1953) is given below.

Theorem 7: Let $A$ and $B$ be constant matrices and $C(t)$ and $D(t)$ be matrices whose coefficients are measurable functions of $t$ and

$$
\begin{aligned}
& \|C(t)\| \leqslant \tau<1 /\left\|A^{-1}\right\|, \\
& \int_{0}^{\infty}\left\|C^{\prime}(t)\right\| \mathrm{d} t<\infty, \\
& \int_{0}^{\infty}\|D(t)\| \mathrm{d} t<\infty .
\end{aligned}
$$

Then if all solutions of the system

$$
A x^{\prime}=B x \text {, where } A \text { is nonsingular, }
$$

are bounded in $[0, \infty)$, then all solutions of $(14)$,

$$
[A+C(t)] x^{\prime}=[B+D(t)] x,
$$

are also bounded in $[0, \infty)$.

A problem of great practical importance is that of small periodic perturbations. The perturbations may be due to uncertainties in modelling, or due to disturbances, or both. The following theorem deals with systems of this type.

Theorem 8: Let $A$ and $B$ be constant matrices and $C(t)$ and $D(t)$ be matrices whose elements are continuous periodic functions of $t$ with period $T$. If all solutions of the system (18), 


$$
A x^{\prime}=B x \text {, where } A \text { is nonsingular, }
$$

approach zero as $t \rightarrow \infty$, then for small values of $\varepsilon(\|\varepsilon\| \ll 1)$, all solutions of

$$
[A+\varepsilon C(t)] x^{\prime}=[B+\varepsilon D(t)] x,
$$

approach zero as $t \rightarrow \infty$.

According to this theorem, if the perturbations are sufficiently small, then the solutions of the perturbed system approach zero provided the solutions of the unperturbed system approach zero as $t$ tends to infinity.

\section{Input-output stability}

In the last two sections, results on the internal stability (stability in the absence of external forcing functions) of systems were described. In the complete stability analysis of a physical system, both the internal and the external (stability in the presence of forcing functions) stability are important. This task is simplified to a great extent in LTI systems because internal and external stability are related. Unfortunately, this relationship does not carry over to LTV systems and hence external and internal stability have to be ascertained separately. Results presented in this section on external stability (or input-output stability) were derived using the methods of functional analysis.

The existing methods of analysis (Desoer \& Vidyasagar 1975) deal with firstorder systems of the form (2),

$$
x^{\prime}(t)=B(t) x(t)+R(t) v(t) .
$$

Application of these results to multidimensional second-order systems is possible only after conversion of the second-order system to first-order, which, as mentioned earlier, poses serious practical difficulties. Hence it is desirable to have results directly for the second-order system, or at least for the modified first-order form (3),

$$
A(t) x^{\prime}(t)=B(t) x(t)+R(t) v(t) .
$$

Equations containing arbitrarily time-varying operators are difficult to handle. It has been shown that all linear time invariant operators that satisfy some slight continuity properties have a convolutional representation. It is much easier to study this than the general case which does not admit a convolutional representation. Consequently, in most of the analysis on input-output stability, the arbitrarily varying system is split into an LTI system in the forward path and a time varying linear system in the feedback path. Hence the second-order system to be treated is of the form

$$
\left[M_{1}+M_{2}(t)\right] q^{\prime \prime}(t)+\left[G_{1}+G_{2}(t)\right] q^{\prime}(t)+\left[K_{1}+K_{2}(t)\right] q(t)=P(t) w(t) .
$$

In order to analyse it as such, $\mathscr{L}^{-1}\left\{\left(s^{2} M_{1}+s G_{1}+K_{1}\right)^{-1}\right\}$ must be found. As a closed form solution does not exist for this expression, the authors were forced to analyse

$$
[A+C(t)] x^{\prime}(t)=[B+D(t)] x(t)+R(t) v(t) .
$$

However, this does not pose serious practical difficulties as the second-order form 
can be easily converted into this form. Theorems on both $L_{2}$ and $L_{\infty}$ stabilities are described. The first part of this section deals with $L_{2}$ stability. In the following, we denote $R(t) v(t)$ by $u(t)$. The main theorem (Pradeep 1987; Pradeep \& Shrivastava 1987b) on $L_{2}$ stability reads as follows.

Theorem 9: Let $B$ denote a constant $(n \times n)$ matrix, all of whose eigenvalues have negative real parts. Let $C(t)$ and $D(t)$ denote $(n \times n)$ matrix-valued functions of $t$, whose elements are measurable and uniformly bounded for $t \geqslant 0$; also, the elements of $C$ are differentiable. Let $x$ be a complex $n$-vector-valued function of $t$ defined and differentiable on $[0, \infty)$ such that

$$
[I+C(t)] x^{\prime}(t)=[B+D(t)] x(t)+u(t),
$$

with $u \in L_{2}^{n}[0, \infty)$.

Let $\xi_{j}+i n_{j}(j=1, \ldots, n)$ be the eigenvalues of $B$.

Then, let $\xi \triangleq \xi_{m}$, where $\left|\xi_{m}\right|=\min _{j}\left|\xi_{j}\right|$.

If

$$
\begin{aligned}
& \|C(t)\| \leqslant c<1, \text { and } \\
& c+(1 /|\xi|) \sup _{t \geqslant 0} \Lambda\left\{D(t)+C^{\prime}(t)-B C(t)\right\}<1,
\end{aligned}
$$

then $x \in L_{2}^{n}[0, \infty)$ and $x(t) \rightarrow 0$ as $t \rightarrow \infty$.

The determination of the largest eigenvalue of the matrix $\left\{D(t)+C^{\prime}(t)-B C(t)\right\}$ is not an easy task. However, it may be simplified considerably by exploiting the inequality (Sandberg 1965),

$$
\Lambda\{A\} \leqslant n \max _{j, k}\left|a_{j k}\right| .
$$

Several other inequalities also exist in literature which place bounds on the eigenvalues of a matrix.

Though the terminology used in this theorem and the theorems and corollaries that follow may look a little abstract to the practising engineer, they are easy to apply. Most complex engineering systems are well-behaved mathematically; the elements of the matrices $C$ and $D$ are generally measurable, uniformly bounded and differentiable. It is easy to verify that the constant matrix $B$ has negative eigenvalues, and verification of (23) may be greatly simplified as mentioned above. Hence the application of the theorem is easy and straightforward.

A corollary of theorem 9 dealing with a more general form of the governing equation,

$$
[A+C(t)] x^{\prime}(t)=[B+D(t)] x(t)+u(t)
$$

has also been derived (Pradeep \& Shrivastava 1987b). Another corollary of this theorem deals with constant coefficient systems subjected to small periodic perturbations. This corollary states -

Corollary 9.1 Let $B$ denote a constant $(n \times n)$ matrix all of whose eigenvalues have negative real parts. Let $C(t)$ and $D(t)$ denote $(n \times n)$ periodic matrix-valued functions of $t$, whose elements are measurable. In addition, the elements of $C$ are differentiable. Let $x$ be a complex $n$-vector-valued function of $t$ defined and 
differentiable on $[0, \infty)$ such that

$$
[l+\varepsilon C(t)] x^{\prime}(t)=[B+\varepsilon D(t)] x(t)+u(t),
$$

with $u \in L_{2}^{n}[0, \infty)$ and $\varepsilon \ll 1$.

Let $\xi_{j}+i n_{j}(j=1, \ldots n)$ be the eigenvalues of $B$.

Then, let $\xi \triangleq \xi_{m}$ where $\left|\xi_{m}\right|=\min _{j}\left|\xi_{j}\right|$.

If $\xi>O(\varepsilon)$, then $x \in L_{2}^{n}[0, \infty)$ and $x(t) \rightarrow 0$ as $t \rightarrow \infty$.

Here, $\xi>O(\varepsilon)$ if $\xi=O\left(\varepsilon^{n}\right)$ where $n>1$.

The conditions in this corollary are very easy to verify.

For practical applications, $L_{2}$ stability is sometimes restrictive. In many physical systems, $L_{\infty}$ stability, i.e., a bounded output for a bounded input, is more important. Conditions for $L_{\infty}$ stability of the modified first-order systems are described in the following. The main theorem (Pradeep 1987; Pradeep \& Shrivastava 1987c) is given below.

Theorem 10: Let $B$ denote a constant $(n \times n)$ matrix. Let there exist a $c_{1}>0$ such that all the eigenvalues of $\left(B+c_{1} I\right)$ have negative real parts. Let $C(t)$ and $D(t)$ denote $(n \times n)$ matrix-valued functions of $t$, whose elements are measurable and uniformiy bounded for $t \geqslant 0$. In addition, the elements of $C$ are differentiable. Let $x$ be a complex $n$-vector-valued function of $t$, defined and differentiable on $[0, \infty)$, such that

$$
[I+C(t)] x^{\prime}(t)=[B+D(t)] x(t)+u(t),
$$

where $e^{c_{1} t} u(t) \in L_{2}^{n}[0, \infty)$.

Let $\xi_{j}+i n_{j}(j=1, \ldots, n)$ be the eigenvalues of $B$.

Then, let $\xi=\xi_{m}$ where $\left|\xi_{m}\right|=\min _{j}\left|\xi_{j}\right|$.

If $\|C(t)\| \leqslant c<1$, and

$$
c+\frac{1}{\left|\xi-c_{1}\right|} \sup _{t>0} \Lambda\left\{D(t)+C^{\prime}(t)-B C(t)\right\}<1,
$$

then there exist $c_{2}, c_{3}, c_{4}$, all positive, such that $x \in L_{\infty}^{n}[0, \infty)$ and

$$
\|x(t)\| \leqslant \frac{1}{(1-c)} \cdot\left[c_{2} e^{-c_{1} t}+c_{3}+c_{4} \int_{0}^{t}\|u(\tau)\| \mathrm{d} \tau\right], \text { for } t \geqslant 0 .
$$

A corollary of theorem 10, dealing with (24) has been derived. As in the case of $L_{2}$ stability, a corollary dealing with constant coefficient systems subjected to periodic perturbations has also been derived.

Corollary 10:1: Let $B$ denote a constant $(n \times n)$ matrix. Let there exist a $c_{1}>0$ such that all the eigenvalues of $\left(B+c_{1} I\right)$ have negative real parts. Let $C(t)$ and $D(t)$ denote $(n \times n)$ real periodic matrix-valued functions of $t$ whose elements are measurable. In addition, the elements of $C$ are differentiable. Let $x$ be a complex $n$ vector-valued function of $t$ defined and differentiable on $[0, \infty)$ such that

$$
[I+\varepsilon C(t)] x^{\prime}(t)=[B+\varepsilon D(t)] x(t)+u(t),
$$


with $e^{c_{1} t} u(t) \in L_{2}^{n}[0, \infty)$ for $\varepsilon \ll 1$.

Let $\xi_{j}+i n_{j}(j=1, \ldots, n)$ be the eigenvalues of $B$.

Then, let $\xi=\xi_{m}$ where $\left|\xi_{m}\right|=\min _{j}\left|\xi_{j}\right|$,

If $\|C(t)\| \leqslant c$ and $\left|\xi-c_{1}\right|>O(\varepsilon)$, then

$x \in L_{\propto}^{n}[0, \infty)$ and there exist positive constants $c_{2}, c_{3}, c_{4}$ such that

$$
\|x(t)\|<\frac{1}{(1-\varepsilon c)}\left[c_{2} e^{-c_{1} t}+c_{3}+c_{4} \int_{0}^{1}\|u(\tau)\| \mathrm{d} \tau\right], \text { for } t \geqslant 0 .
$$

Several theorems on the stability of linear multidimensional systems have been stated. The theorems were derived with the needs of the practising engineer in mind; lengthy mathematical derivations may not appeal to him; he needs solid proof for the utility of the theorems. Here an effort has been made to find conditions as far as possible on the physical system parameters directly. Also as an illustration, it has been shown (Pradeep 1987; Pradeep \& Shrivastava 1987d) that, for the damped Mathieu equation, the stability region obtained by using theorem 2 is superior to the existing results (Gunderson et al 1974; Taylor \& Narendra 1969) for some range of parameter values. In another such illustration, the asymptotic behaviour of the planar motion of an arbitrarily shaped satellite with a damper assembly in an elliptic orbit is analysed (Modi \& Neilson 1969). The attitude dynamics of a rigid, axisymmetric, spinning satellite in an eccentric orbit subjected to gravity gradient torque is analysed for both $L_{2}$ and $L_{\infty}$ stabilities (Modi \& Brereton 1968).

Two other examples that have been analysed are (i) the stability of an extending pendulum, and (ii) the stability of lateral vibrations of an extending cantilever rod (Jankovic 1976). These examples are of great practical importance as they provide an understanding of the deployment dynamics of space structures and tethers.

\section{Conclusion}

This paper presents the statements of some theorems on the stability of multidimensional linear systems with time varying parameters, derived by the authors during a recent investigation. The detailed proofs and illustrations are available in other papers. The emphasis throughout the course of the investigation has been on the practical applicability of results. It is expected that the results would turn out to be of considerable use to practising engineers dealing with rather complex systems. The theorems are applicable to a wide range of systems in different disciplines.

The first author is grateful to the trustees of the $\mathrm{J} N$ Tata Endowment for financial support.

\section{References}

Bellman R 1943 Duke Math. J. 10: 643-647

Bellman R 1953 Stability theory of differential equations (New York: McGraw-Hill) 
Cesari L 1963 Asymptotic behaviour and stability problems in ordinary differential equations (New York: Springer-Verlag)

Coppel W A 1965 Stability and asymptotic behaviour of differential equations (Boston: Heath)

Desoer C A, Vidyasagar M 1975 Feedback systems: Input-output properties (New York: Academic Press) Gronwall T H 1918 Ann. Math. 20: 212-296

Gunderson H, Rigas H, Van Vleck F S 1974 SIAM J. Appl. Math. 26: 345-349

Huseyin K 1978 Vibrations and stability of multiple parameter systems (Netherlands: Sijthoff \& Noordhoff)

Jankovic M S 1976 Lateral vibrations of an extending cantilever rod. University of Toronto, Institute for Aerospace Studies, Technical Note 202

Kreider D L, Kuller R G, Ostberg D R 1968 Elementary differential equatians (Massachusetts: Addison Wesley)

Lancaster P 1966 Lambda-matrices and vibrating systems (New York: Pergamon)

Modi V J, Brereton R C 1969 CASI Trans. (Can. Aeronaut. Space Inst.) 2: 44-48

Modi V J, Neilson J E 1968 Aeronaut. J, 72: 807-810

Pradeep S 1987 On the stability of multidimensional linear systems with time varying parameters, Ph.D. dissertation, Indian Institute of Science, Bangalore

Pradeep S, Shrivastava S K 1987a Acta Astronaut. (to be published)

Pradeep S, Shrivastava S K 1987 b. Astronaut. Sci. (to be published)

Pradeep S, Shrivastava S K 1987c J. Guidance Control Dyn. (to be published)

Pradeep S, Shrivastava S K 1987d Mech. Res. Commun. (to be published)

Sandberg I W 1965 SIAM J. Control 2: 192-195

Shrivastava S K 1981 J. Appl. Mech. 48: 174-176

Shrivastava S K, Pradeep S 1985 J. Guidance Control Dyn. 8: 579-583

Taylor J H, Narendra K S 1969 SIAM J. Appl. Math. 17: 343-352 